\title{
Testosterone Protects Mice Against Zika Virus Infection And Suppresses The Inflammatory Response In Brain
}

\section{Bohan Zheng}

Huazhong Agricultural University

Jiajun Sun

Huazhong Agricultural University

Haoran Luo

Huazhong Agricultural University

Ling'en Yang

Huazhong Agricultural University

Qi Li

Huazhong Agricultural University

\section{Luping Zhang}

Huazhong Agricultural University

\section{Youhui Si}

Huazhong Agricultural University

\section{Shengbo Cao}

Huazhong Agricultural University

Jing Ye ( $\square$ yej@mail.hzau.edu.cn )

Huazhong Agricultural University https://orcid.org/0000-0002-3258-6224

\section{Research}

Keywords: ZIKV, Testosterone, Neuroinflammation, CD8+ T cells, Treatment

Posted Date: November 23rd, 2021

DOI: https://doi.org/10.21203/rs.3.rs-1084468/v1

License: (c) (i) This work is licensed under a Creative Commons Attribution 4.0 International License.

Read Full License 


\section{Abstract}

Background: Testosterone is an important hormone affecting human growth and development. Recent studies have shown that testosterone is related to immune regulation. Infection with Zika virus (ZIKV) can cause testicular damage and decrease testosterone secretion. However, whether testosterone plays a function in the pathogenesis of ZIKV is still unclear. The main objective of this study was to understand the role of testosterone in central nervous system injury and inflammation induced by ZIKV.

Methods: In this work, a mouse model was used to evaluate the role of testosterone in ZIKV infection. Histopathological analysis, flow cytometry, and real-time PCR were performed to investigate the mechanism by which testosterone improved survival in mice after ZIKV infection.

Results: ZIKV infection caused testicular damage and decreased testosterone secretion in mice, and testosterone supplementation after ZIKV infection reduced mortality and pathological symptoms in mice. Histopathological analysis showed that testosterone treatment after ZIKV infection could reduce inflammatory cell infiltration in the brain and alleviate brain injury. Flow cytometry and quantitative realtime PCR results confirmed that testosterone treatment could reduce the $\mathrm{CD} 8^{+} \mathrm{T}$ cell infiltration and the expression of interferon gamma and inflammatory cytokines induced by ZIKV.

Conclusion: Testosterone plays an important protective role in mice during ZIKV infection and can be used as a potential therapeutic treatment for ZIKV infection.

\section{Background}

ZIKV is a member of Flavivirus which contains a positive-stranded RNA genome that encodes three structural proteins (capsid (C), pre-membrane (prM) and envelope (E)) and seven non-structural proteins (NS1, NS2A, NS2B, NS3, NS4A, NS4B and NS5) [1]. The first isolation of ZIKV was made in April 1947 from the serum of a pyrexial rhesus monkey[2]. In the half-century since its discovery, ZIKV infections have been detected only sporadically, until an outbreak in Yap Island was reported in 2007. About threequarters of Yap residents older than three years of age are infected with ZIKV[3]. ZIKV massive spread into the Americas from 2015[4], and reached at the peak of the ZIKV epidemic in 2016-2017. The number of ZIKV cases in Brazil reached 216,207 and 8,604 babies were born with deformities. [5, 6].

ZIKV has obvious tissue phagocytosis, mainly damage to the nervous system and reproductive system[7, 8]. Of mounting concern are reports linking ZIKV infection to microcephalic births and serious neurological complications, such as Guillain-Barre' syndrome (GBS) which is an acute inflammatory immune-mediated polyradiculoneuropathy characterized by tingling, progressive weakness, autonomic nervous disorder, and pain[9]. Non-vector transmission of ZIKV infection can occur during childbirth (mother-to-child), organ transplantation, blood transfusion, and sexual contact[10]. Recent studies have found that the ZIKV can remain dormant in the male reproductive organs for up to six months[11], which is a potential threat to infected men and their partners. Through the establishment of type-l interferon receptor deficient (A129) mice infection model, the phenomenon of male system damage caused by ZIKV 
infection was revealed. ZIKV causes testicular damage by inducing inflammation of the testis and epididymis. Testosterone levels are elevated in the initial stage of viral infection and it remained at a low level after 7 days of infection[12,13].

Testosterone is a steroid hormone produced by the testicles of men or the ovaries of women. Leydig cells are the main testosterone producing cells[14]. Testosterone affects growth and development and also regulates the body's immune response[15]. Studies have shown that sex hormone differences are the reason for why women are more likely to develop autoimmune diseases than men [16]. For example, in animal studies of systemic lupus erythematosus (SLE), an autoimmune inflammatory disease, female mice treated with testosterone have been found to have reduced disease severity and longer survival[17]. Rheumatoid arthritis (RA) is a systemic disease of articular and extrarticular co-existence caused by an autoimmune response, mainly in women. Additionally, in SKG mice (a mouse model of rheumatoid arthritis), testosterone prevented the development of joint and lung disease in male mice[18]. Furthermore, testosterone affects tumor development through immunosuppression. For instance, in follicular thyroid cancer, testosterone has been shown to influence tumor progression by inhibiting $\mathrm{CD} 8^{+}$ T cells and M1 macrophages[19]. Testosterone also plays an important role in the central nervous system and can improve cognitive performance by improving synaptogenesis[20]. Treatment with testosterone reduces the rate of whole brain atrophy[21] and preserves excitatory synaptic transmission during autoimmune demyelination[22].

In this study, we found that ZIKV infection caused testicular damage and decreased testosterone secretion. Testosterone injection reduced mortality of ZIKV infected mice. Further analysis revealed that testosterone treatment inhibits $\mathrm{CD} 8^{+} \mathrm{T}$ cell activation in the spleen and infiltration in brain. Reduced inflammatory cytokines was also observed in brain tissue. These results suggest that testosterone has a protective effect on mice after ZIKV infection, indicating it may be a potential drug candidate for treatment of ZIKV.

\section{Materials And Methods}

Cell cultures and virus

TM4 (mouse Sertoli cell line) cells, Vero (African green monkey kidney cell line) cells and A549 (human alveolar epithelium cell line) cells were maintained in Dulbecco modified Eagle medium (DMEM; Sigma) supplemented with $10 \%$ fetal bovine serum (FBS), $100 \mu \mathrm{g} / \mathrm{ml}$ streptomycin, and $100 \mathrm{U} / \mathrm{ml}$ penicillin at $37^{\circ} \mathrm{C}$ in a 5\% CO2 atmosphere. ZIKV H/PF/2013 strain (GenBank: KJ776791) was kindly provided by Dr. Bo Zhang, Wuhan Institute of Virology, Chinese Academy of Sciences and was propagated and titrated on Vero cells.

Experiments on animals

All mouse experimental procedures were approved by the Institutional Animal Care and Use Committee of Huazhong Agricultural University, ID Number is HZAUMO-2021-0164. Adult male 8-week-old A129 mice 
were purchased from the Laboratory Animal Center of Huazhong Agricultural University, Wuhan, China. The mice were randomly divided into four groups with six mice in each group. group 1, control group (sesame seed oil); group 2, only testosterone-treated group (testosterone); group 3, ZIKV-infected group (ZIKV); and group 4, ZIKV-infected and testosterone-treated group (ZIKV + testosterone). Mice belonging to the ZIKV and ZIKV + testosterone groups were intraperitoneally injected with $10^{3} \mathrm{PFU}$ of ZIKV $\mathrm{H} / \mathrm{PF} / 2013$ strain in $100 \mu \mathrm{l}$ of DMEM. On the $2 \mathrm{nd}$ and 4 th day after ZIKV infection, the mice belonging to the testosterone and ZIKV + testosterone group were intraperitoneally injected with $0.05 \mathrm{mg}$ testosterone in $100 \mu \mathrm{l}$ of sesame seed oil.

\section{Virus infection}

TM4 cells or A549 cells were plated in 24-well plates. After washing with DMEM, cells were infected with ZIKV H/PF/2013 strain of 0.5MOI. The mock-infected cells were prepared using the same procedures with no virus.

Cell viability assay

CellTiter-Glo® One Solution Assay kit (Promega) was used to measure cell viability. A549 cells and TM4 cells were inoculated in a $96-$ well cell culture plate at a density of $1 \times 10^{5}$ cells $/ \mathrm{mL}$, and incubated at $37^{\circ} \mathrm{C}$ with $5 \% \mathrm{CO} 2$ for $24 \mathrm{~h}$. The supernatants were replaced with testosterone or DMSO of different concentrations, each concentration was measured in triplicates. After $48 \mathrm{~h}$, the cells were washed with phosphate buffered saline (PBS) and 100 $\mathrm{L}$ CellTiter-Glo reagent was added to each well. For proper cell lysis, the cells were stirred in a shaker for $2 \mathrm{~min}$ and then incubated at room temperature for $10 \mathrm{~min}$. The luminescence signal under each condition was quantified using a multi-template reader, and the luminescence values were compared with their corresponding DMSO controls.

RNA extraction and quantitative real-time PCR

Total RNA of cells was extracted individually by Trizol reagent (Invitrogen), and $1 \mu \mathrm{g}$ of RNA was used to synthesize cDNA using a ABscript cDNA First Strand synthesis kit (ABclonal Technology). Quantitative real-time RT-PCR was performed using a 7500 real-time PCR system (Applied Biosystems) and 2X Universal SYBR Green Fast qPCR Mix (ABclonal Technology). Data were normalized to the level of $\beta$-actin expression in each sample. Primers were as follows:

TNF-a-F: 5'-CAGGCGGTGCCTATGTCTC -3',

TNF-a-R: 5'-CGATCACCCCGAAGTTCAGTAG -3';

CCL-5-F: 5'-TGCCCACGTCAAGGAGTATTTC-3',

CCL-5-R: 5'-AACCCACTTCTTCTCTGGGTTG-3';

IL-6-F: 5'- CATGTTCTCTGGGAAATCGTG -3', 
IL-6-R: 5'- TCCAGTTTGGTAGCATCCATC -3';

CCL-2-F: 5'-CGGCGAGATCAGAACCTACAAC-3',

CCL-2-R: 5'-GGCACTGTCACACTGGTCACTC-3';

ß-actin-F: 5'-CACTGCCGCATCCTCTTCCTCCC-3',

ß-actin-R: 5'-CAATAGTGATGACCTGGCCGT-3'.

ZIKV-F: 5'- AACATGGCGGAGGTAAGA-3',

ZIKV-R: 5'- TGTCTGATTGCTTGTCAAGGT-3';

Enzyme-linked immunosorbent assay (ELISA)

The mice brain tissues were homogenized in PBS and centrifuged at $5000 \times \mathrm{g}$ to obtain the supernatant. IFN- $y$ levels in the brain were determined according to the instructions of ELISA kit purchased from ABclonal Technology. The serum was separated by centrifugation at $1000 \times \mathrm{g}$ for $5 \mathrm{~min}$ after the mice blood was placed at $37^{\circ} \mathrm{C}$ for $30 \mathrm{~min}$. Serum testosterone levels were measured using an ELISA kit purchased from abcam.

Flow cytometry

The mice spleens were separated and added to PBS and ground with a $40 \mu \mathrm{m}$ cell strainer (FALCON). After centrifugation at $1600 \mathrm{r} / \mathrm{min}$ at $4^{\circ} \mathrm{C}$ for $5 \mathrm{~min}$, supernatant was discarded and red blood cell lysate was added to resuspend cells. The lysis was terminated by PBS and centrifuged. The supernatant was discarded and the cells were suspended by $0.2 \% \mathrm{BSA} .10^{6}$ cells were removed for staining. Antibodies CD3-FITC, CD4-PE, and CD8-APC were purchased from BD BioSciences. Cell nuclei were stained with 6diamidino-2-phenylindole (DAPI; Invitrogen) to distinguish dead cells. The number of $\mathrm{CD}^{+} \mathrm{T}$ cells was detected and analyzed by flow cytometry.

\section{Statistical analysis}

All experiments were conducted at least three times under similar conditions. The resulting data was analyzed using GraphPad Prism version 5. The results were expressed by mean \pm standard error (SEM) and median. Two-way analysis of variance (ANOVA) with subsequent tests were used for multiple comparisons using Bonferroni posttest to determine statistical differences between the experimental groups. In all tests, a p values of $<0.05$ was considered significant.

\section{Results}

ZIKV infection causes testicular damage and testosterone decline in mice 
ZIKV has obvious organization preferences, and the previous studies have shown that the brain and testicles are the main target organs of the virus in male mice $[12,23]$. To study the effect of ZIKV on testicular injury, the male A129 mice were pathologically analyzed at 8 days after ZIKV infection. Compared with the control group, the vas deferens and testicles were obviously congested (Fig. 1A-B). The seminiferous tubules of ZIKV infected mice were lost the original structure, and spermatogenic cells arranged loose and mussily (Fig. 1C). Testosterone is mainly produced by the testes. To test whether testicular damage caused by ZIKV infection could affect testosterone secretion, blood samples were taken from the tail of mice on day 8 post ZIKV infection to detect serum testosterone concentration, and testicles were ground to detect testosterone concentration in testicles. The results showed that ZIKV infection reduced the levels of testosterone in serum and testis (Fig. 1D-E).

Testosterone supplementation decreases ZIKV-induced mouse lethality

Previous studies have shown that ZIKV can enter the brain of mice and cause severe inflammation[25] while testosterone has an immunosuppressive effect. To investigate whether testosterone plays a protective role during ZIKV infection, ZIKV-infected A129 mice were injected with testosterone or the same dose of sesame oil at the second day and third day after ZIKV infection (Fig. 2A). On day 8 post infection, blood samples were collected to detect the testosterone concentration. As shown in the results, testosterone was maintained at a higher level in the ZIKV-infected and testosterone-treated group compared with the ZIKV-infected and sesame oil-treated group (Fig. 2B). All mice in the testosterone or sesame oil-treated group survived during the observation, while mice in the ZIKV-infected and sesame oil treated or testosterone-treated group started to display morbidity and mortality on day 9 or 10 postinfection. After ZIKV infection, a high mortality rate (83\%) of mice was observed in the sesame oil group, while the mortality rate of mice in testosterone treated group was $50 \%$ (Fig. $2 \mathrm{C}$ ). In addition, treatment with testosterone caused fewer declines in body weight (Fig. 2D) and improved behavioral signs in ZIKVinfected mice (Fig. 2E). These results suggest that testosterone treatment after ZIKV infection can alleviate the symptoms and increase the survival rate of mice, revealing that testosterone plays a protective role on mice infected with ZIKV.

Testosterone treatment reduces ZIKV-induced inflammatory response in mouse brain tissues

Brain is one of the main target organs for ZIKV, which can invade the brain of both fetuses and adults and cause encephalitis, myelitis and meningoencephalitis [26, 27]. Given the role of testosterone in reducing the ZIKV-induced mouse lethality, we investigated whether testosterone can eliminate the neuroinflammation caused by ZIKV infection. A129 mice were injected with testosterone or sesame oil following ZIKV infection. H\&E staining was performed for histopathological changes analysis of the brain sections on the eighth day after virus infection. Results showed the signatures of perivascular cuffing in ZIKV-infected mice at 8 days post infection, whereas these indicators of encephalitis were reduced in ZIKV-infected mice receiving testosterone treatment (Fig. 3A). In addition, testosterone treatment can reduce meningitis (Fig. 3B) and haemorrhage in the hippocampus (Fig. 3C) caused by ZIKV infection. Furthermore, RT-qPCR results showed that testosterone treatment reduced the expression of 
proinflammatory factors IL- 6 and TNF-a induced by ZIKV infection (Fig. 3D-E). Taken together, these results demonstrate that testosterone treatment reduced inflammation and pathological changes caused by ZIKV infection in brains of mice.

Testosterone treatment does not affect the viral replication and humoral immunity in ZIKV infected mice

To investigate whether testosterone protects mice and alleviate the neuroinflammation by interfering with viral replication, A549 cells and TM4 cells were used to detect the in vitro replication of ZIKV. The cytotoxicity of testosterone on A549 cells and TM4 cells was examined by cell viability assay. Viable cells were determined on the basis of ATP quantification of cells, which indicates the presence of metabolically active cells. Different concentrations of testosterone were added on A549 cells and TM4 cells, and the results revealed that $10 \mu \mathrm{M}$ testosterone exhibits no cytotoxic effect, while a $100 \mu \mathrm{M}$ concentration was significantly toxic to cells (Fig. 4A). A concentration of $10 \mu \mathrm{M}$ was then used to test the effect of testosterone on viral replication. A549 cells and TM4 cells were treated with $10 \mu \mathrm{M}$ testosterone followed by ZIKV infection. At 24, 36, and 48 hours post infection, cells were collected to detect the RNA level of and viral titer of ZIKV. The results showed that testosterone did not inhibit the replication of ZIKV (Fig. 4B-C).

Since testosterone does not affect the replication of the virus, we detected the antibody level in the serum of ZIKV infected mice to explore whether testosterone protected the mice by increasing the antibody level. Serum samples were collected on 8 days after ZIKV infection, and the antibody against ZIKV E protein was detected by ELISA. No significant change of antibody level was observed upon testosterone treatment in mice (Fig. 4D). These results suggest that testosterone treatment does not play protective role on ZIKV infection by interfering with the viral replication and humoral immunity.

Testosterone inhibits T cell activation caused by ZIKV infection

To further investigate how testosterone participate the pathogenic process of ZIKV infection, we analyzed the proportion of $\mathrm{CD} 4^{+} \mathrm{T}$ cells and $\mathrm{CD} 8^{+} \mathrm{T}$ cells in spleen of mice by flow cytometry. The results showed that ZIKV infection led to a significant increase in the proportion of $\mathrm{CD}^{+} \mathrm{T}$ cells in spleen, while testosterone injection could reduce the increased $C D 3^{+} T$ cells ratio caused by ZIKV infection (Fig. 5A-B). Although testosterone treatment did not significantly change the proportion of $\mathrm{CD} 8^{+} \mathrm{T}$ cells in $\mathrm{CD} 3^{+} \mathrm{T}$ cells, it significantly reduced the proportion of $C D 8^{+} T$ cells in spleen cells (Fig. 5A, C-D). However, infection with ZIKV did not change the proportion of $\mathrm{CD}^{+}{ }^{+} \mathrm{T}$ cells in the spleen (Fig. 5A, E-F).

Testosterone treatment reduces the infiltration of $\mathrm{CD} 8^{+} \mathrm{T}$ cells in brain of mice infected with ZIKV

ZIKV infection can cause $\mathrm{CD} 8^{+} \mathrm{T}$ cells to enter the brain and produce an inflammatory response[28]. Here to determine the effect of ZIKV infection and testosterone supplementation on infiltration of $\mathrm{CD}^{+} \mathrm{T}^{\mathrm{T}}$ cells in mouse brain, the immunohistochemical assay was performed to analyze the $\mathrm{CD} 8^{+} \mathrm{T}$ cell numbers. More $\mathrm{CD} 8^{+} \mathrm{T}$ cells in the ZIKV infected mouse brain than those in the uninfected mouse brain was 
observed, while treatment of testosterone reduced the $\mathrm{CD} 8^{+} \mathrm{T}$ cell infiltration caused by ZIKV infection (Fig. 6A). To further verify the result, the brain cells in mice were isolated for flow cytometry assay (Fig. 6B). Consistently, the results showed that $C D 8^{+} T$ cells increased significantly after ZIKV infection in mice, while testosterone supplementation reduced the $C D 8^{+} T$ cell number (Fig. $6 C$-D). These results suggest that testosterone treatment could reduce the infiltration of $\mathrm{CD}^{+} \mathrm{T}$ cells in the brain of ZIKV infected mice.

Testosterone treatment reduces IFN- $\gamma$ and chemokine expression in brain of ZIKV infected mice

IFN- $y$ is mainly expressed by $\mathrm{T}$ cells, and previous studies have reported that testosterone can inhibit IFNY secretion signaling pathway[29], so we detected the expression of IFN- $y$ in mouse serum and brain. Mice were sacrificed and blood was collected on day 8 after infection with ZIKV, and their brains were subjected to grind and centrifugation to separate the supernatant. IFN- $y$ levels in brain and serum of mice were detected by ELISA kit. The results showed that the level of IFN-y in the brain was significantly increased after ZIKV infection, and the increase was inhibited by testosterone treatment (Fig. 7A). However, the level of IFN-y in serum was not significantly changed after ZIKV infection (Fig. 7B). Given that chemokines play important role in directing T cell trafficking, the mRNA levels of chemokines, such as CCL2 and CCL5, in mouse brain were analyzed by real-time RT-PCR. It was found that testosterone inhibited the upregulation of chemokines caused by ZIKV infection (Fig. 7C-D).

\section{Discussion}

ZIKV infection usually leads to mild clinical symptoms, but some patients also develop inflammation in central nervous system, leaving neurological sequelae [30]. In this study, we found severe inflammation in brain of mice infected with ZIKV. Infiltration of activated CD8 ${ }^{+} \mathrm{T}$ cells into the brain may be one of the main causes. $C D 8^{+} T$ cells are important immune cells that can either release inflammatory factors or kill target cells directly. Previous studies have reported that ZIKV-specific CD8 ${ }^{+} \mathrm{T}$ cells can prevent ZIKV infection in the central nervous system[28]; however, excessive CD ${ }^{+} \mathrm{T}$ cells entering the brain can lead to segmental apoptotic neurodegeneration, astrocyte proliferation and microglial activation, resulting in encephalitis and even death of the body [31, 32]. In this study, neurologic symptoms such as hind limb paralysis were observed in mice infected with ZIKV, but whether it is related to infiltration of CD8 ${ }^{+} \mathrm{T}$ cells in brain is still needed for further study.

Inflammation is a specific function of the immune system that can resist the invasion of pathogens; however, some pathogens cause an excessive storm of inflammation in the immune system that can damage the body. After SARS-CoV-2 infection, macrophages and monocytes are recruited in response to infection, releasing cytokines and initiating adaptive $T$ and $B$ cell immune responses. This process usually clears viral infections, but in some patients, a dysfunctional immune response occurs, triggering a cytokine storm that mediates widespread lung inflammation[33]. The results showed higher blood plasma levels of IL-2, IL-7, IL-10, IP-10, MCP1, and TNF-a were significantly increased in patients with 
severe COVID-19[34]. Therefore, suppression of inflammation caused by viral infection is an important direction in the treatment of COVID-19[35]. ZIKV infection can also lead to inflammation of the brain, resulting in sequelae and even death of the host. Regulating the intensity of inflammation may also be used as a treatment after ZIKV infection.

Multiple studies have found that there is a gap between men and women in immune response, for example, women are more prone to autoimmune diseases such as Systemic lupus erythematosus (SLE) and Rheumatoid arthritis (RA) $[17,36]$. And the reason for its occurrence is likely to be caused by differences in sex hormones. Through the gender statistics of ZIKV patients, it was found that the female infection is more than the male[37]. Now, there are two possible explanations for this. One is that ZIKV can be sexually transmitted from male to female, resulting in an increase in the number of female patients. The other one is that female patients tend to seek medical treatment due to pregnancy and other factors, resulting in an increase in the number of statistics. Based on the above studies, we speculated that different sex hormones may also lead to more female patients with ZIKV than male patients, and supplementing or antagonizing hormones could be used as a strategy to regulate immune responses, treat autoimmune diseases or inhibit virus-induced inflammatory storms.

Testosterone, a sex hormone produced by the body, has been used to treat sexual dysfunction and osteoporosis in both men and women[38,39]. The advantage of treatment with testosterone over other drugs is its safety in humans [40]. In recent years, many studies on the treatment of testosterone have been reported, mainly because testosterone can inhibit interferon, inflammatory factors and other immune responses [41, 42]. Although the immune response is designed to protect the body from pathogens, excessive inflammation can cause damage to the body itself, and the use of testosterone may play a role in balancing the resistance to pathogens and the damage to the body itself.

In our study, testosterone was found to protect mice against ZIKV infection. Testosterone injection after ZIKV infection increased survival by 33 percent and resulted in attenuated inflammatory response in brain of mice compared with untreated mice. The protection efficiency of testosterone in mice infected with ZIKV may be further improved by exploring the time and method of treatment. In addition, whether testosterone injection can protect hypogonadal patients or female patients with ZIKV infection deserves further exploration.

\section{Conclusions}

This study provides evidence that testosterone plays an important role in ZIKV - induced neuroinflammatory response. Our in vitro and in vivo experiments have shown that testosterone treatment possess protective effect on mice against ZIKV infection by reducing $\mathrm{CD} 8^{+} \mathrm{T}$ cells and thereby reducing IFN-y and inflammatory factors in mouse brain. These results indicate that testosterone may be used as a potential drug for the treatment of ZIKV infection.

\section{Abbreviations}


ZIKV: Zika virus

IL: Interleukin

ELISA: Enzyme-linked immunosorbent assay

TNF-a: Tumor necrosis factor- $a$

CCL2: Monocyte chemotactic protein 1

CCL5: Chemokine (C-C motif) ligand 5

IFN-ץ: Interferon-ү

DAPI: 4',6-Diamidino-2-phenylindole

SLE: Systemic lupus erythematosus

FBS: Fetal bovine serum

RA: Rheumatoid arthritis

GBS: Guillain-Barre' syndrome

DMSO: Dimethyl sulfoxide

\section{Declarations}

\section{Ethics approval and consent to participate}

No human data or tissues were used in this study. All mouse experimental procedures were approved by the Institutional Animal Care and Use Committee of Huazhong Agricultural University, ID Number is HZAUMO-2021-0164.

\section{Consent for publication}

Not applicable.

\section{Availability of data and materials}

The data sets used and analyzed during the current study are available from the corresponding author on reasonable request.

\section{Competing interests}

The authors declare no conflicts of interest. 


\section{Funding}

This work was supported by the National Natural Science Foundation of China $(31825025,32022082$, 32030107, and 31972721) and the Natural Science Foundation of Hubei Province (2019CFA010).

\section{Authors' contributions}

ZBH, CSB, SYH, and YJ designed research; ZBH, SJJ, LHR, and YLE performed experiments; ZBH, LQ, and ZLP analyzed data; ZBH and SJJ organized the data; ZBH, CSB, SYH and YJ wrote the paper. All authors read and approved the final manuscript.

\section{Acknowledgements}

We thank Dr. Bo Zhang, Wuhan Institute of Virology, Chinese Academy of Sciences for kindly providing the ZIKV H/PF/2013 strain.

\section{References}

1. Pierson, T.C. and M.S. Diamond, The emergence of Zika virus and its new clinical syndromes. Nature, 2018. 560(7720): p. 573-581.

2. Dick, G.W.A., S.F. Kitchen, and A.J. Haddow, Zika Virus (I). Isolations and serological specificity. Transactions of The Royal Society of Tropical Medicine and Hygiene, 1952. 46(5): p. 509-520.

3. Lanciotti, R.S., et al., Genetic and serologic properties of Zika virus associated with an epidemic, Yap State, Micronesia, 2007. Emerging infectious diseases, 2008. 14(8): p. 1232-1239.

4. Krow-Lucal, E.R., et al., Association and birth prevalence of microcephaly attributable to Zika virus infection among infants in Paraiba, Brazil, in 2015-16: a case-control study. The Lancet Child \& Adolescent Health, 2018. 2(3): p. 205-213.

5. Bogoch, I.I., et al., Anticipating the international spread of Zika virus from Brazil. The Lancet, 2016. 387(10016): p. 335-336.

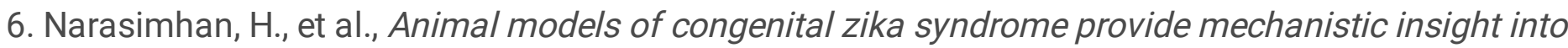
viral pathogenesis during pregnancy. PLoS Negl Trop Dis, 2020. 14(10): p. e0008707.

7. Shahrizaila, N., H.C. Lehmann, and S. Kuwabara, Guillain-Barré syndrome. The Lancet, 2021. 397(10280): p. 1214-1228.

8. Stassen, L., et al., Zika Virus in the Male Reproductive Tract. Viruses, 2018. 10(4).

9. Rodriguez, Y., et al., Guillain-Barre syndrome, transverse myelitis and infectious diseases. Cell Mol Immunol, 2018. 15(6): p. 547-562.

10. Kazmi, S.S., et al., A review on Zika virus outbreak, epidemiology, transmission and infection dynamics. J Biol Res (Thessalon), 2020. 27: p. 5.

11. Barzon, L., et al., Infection dynamics in a traveller with persistent shedding of Zika virus RNA in semen for six months after returning from Haiti to Italy, January 2016. Eurosurveillance, 2016. 
21(32): p. 30316.

12. Ma, W., et al., Zika Virus Causes Testis Damage and Leads to Male Infertility in Mice. Cell, 2016. 167(6): p. 1511-1524.e10.

13. Govero, J., et al., Zika virus infection damages the testes in mice. Nature, 2016. 540(7633): p. 438442.

14. Hall, P.F., D.C. Irby, and D.M.D. Kretser, Conversion of Cholesterol to Androgens by Rat Testes: Comparison of Interstitial Cells and Seminiferous Tubules. Endocrinology, 1969. 84(3): p. 488-496.

15. Trigunaite, A., J. Dimo, and T.N. Jorgensen, Suppressive effects of androgens on the immune system. Cell Immunol, 2015. 294(2): p. 87-94.

16. Whitacre, C.C., Sex differences in autoimmune disease. Nat Immunol, 2001. 2(9): p. 777-80.

17. Roubinian, J.R., R. Papoian, and N. Talal, Androgenic hormones modulate autoantibody responses and improve survival in murine lupus. J Clin Invest, 1977. 59(6): p. 1066-70.

18. Keith, R.C., et al., Testosterone is protective in the sexually dimorphic development of arthritis and lung disease in SKG mice. Arthritis and rheumatism, 2013. 65(6): p. 1487-1493.

19. Zhang, L.J., et al., Testosterone regulates thyroid cancer progression by modifying tumor suppressor genes and tumor immunity. Carcinogenesis, 2015. 36(4): p. 420-8.

20. Frye, C.A., et al., 5alpha-reduced androgens may have actions in the hippocampus to enhance cognitive performance of male rats. Psychoneuroendocrinology, 2004. 29(8): p. 1019-27.

21. Sicotte, N.L., et al., Testosterone treatment in multiple sclerosis: a pilot study. Arch Neurol, 2007. 64(5): p. 683-8.

22. Ziehn, M.O., et al., Therapeutic testosterone administration preserves excitatory synaptic transmission in the hippocampus during autoimmune demyelinating disease. J Neurosci, 2012. 32(36): p. 12312-24.

23. Christian, K.M., H. Song, and G.-L. Ming, Pathophysiology and Mechanisms of Zika Virus Infection in the Nervous System. Annual review of neuroscience, 2019. 42: p. 249-269.

24. Lazear, H.M., et al., A Mouse Model of Zika Virus Pathogenesis. Cell host \& microbe, 2016. 19(5): p. $720-730$.

25. Figueiredo, C.P., et al., Zika virus replicates in adult human brain tissue and impairs synapses and memory in mice. Nature communications, 2019. 10(1): p. 3890-3890.

26. Carteaux, G., et al., Zika Virus Associated with Meningoencephalitis. New England Journal of Medicine, 2016. 374(16): p. 1595-1596.

27. de Almeida Oliveira Evangelista, G., et al., Meningoencephalitis associated with Zika virus and Chikungunya virus infection. Japanese Journal of Infectious Diseases, 2021. advpub.

28. Huang, H., et al., CD8(+) T Cell Immune Response in Immunocompetent Mice during Zika Virus Infection. J Virol, 2017. 91(22).

29. Ho, C.H., et al., Testosterone suppresses uropathogenic Escherichia coli invasion and colonization within prostate cells and inhibits inflammatory responses through JAK/STAT-1 signaling pathway. 
PLoS One, 2017. 12(6): p. e0180244.

30. Barbi, L., et al., Prevalence of Guillain-Barre syndrome among Zika virus infected cases: a systematic review and meta-analysis. Braz J Infect Dis, 2018. 22(2): p. 137-141.

31. Melzer, N., S.G. Meuth, and H. Wiendl, CD8+ T cells and neuronal damage: direct and collateral mechanisms of cytotoxicity and impaired electrical excitability. Faseb j, 2009. 23(11): p. 3659-73.

32. Pitsch, J., et al., CD8(+) T-Lymphocyte-Driven Limbic Encephalitis Results in Temporal Lobe Epilepsy. Ann Neurol, 2021. 89(4): p. 666-685.

33. Tay, M.Z., et al., The trinity of COVID-19: immunity, inflammation and intervention. Nat Rev Immunol, 2020. 20(6): p. 363-374.

34. Huang, C., et al., Clinical features of patients infected with 2019 novel coronavirus in Wuhan, China. Lancet (London, England), 2020. 395(10223): p. 497-506.

35. Yuin Ho, J.S., et al., Topoisomerase 1 inhibition therapy protects against SARS-CoV-2-induced inflammation and death in animal models. bioRxiv: the preprint server for biology, 2020:

p. 2020.12.01.404483.

36. Pikwer, M., et al., Association between testosterone levels and risk of future rheumatoid arthritis in men: a population-based case-control study. Annals of the Rheumatic Diseases, 2014. 73(3): p. 573.

37. Bastos, M.M. and F.C. Coelho, Estimating under-observation and the full size of the 2016 Zika epidemic in Rio de Janeiro. PLoS One, 2018. 13(10): p. e0205001.

38. Golds, G., D. Houdek, and T. Arnason, Male Hypogonadism and Osteoporosis: The Effects, Clinical Consequences, and Treatment of Testosterone Deficiency in Bone Health. Int J Endocrinol, 2017. 2017: p. 4602129.

39. Ingram, C.F., et al., Testosterone therapy and other treatment modalities for female sexual dysfunction. Current Opinion in Urology, 2020. 30(3): p. 309-316.

40. Salter, C.A. and J.P. Mulhall, Guideline of guidelines: testosterone therapy for testosterone deficiency. BJU Int, 2019. 124(5): p. 722-729.

41. Vom Steeg, L.G., et al., Androgen receptor signaling in the lungs mitigates inflammation and improves the outcome of influenza in mice. PLoS Pathog, 2020. 16(7): p. e1008506.

42. Tuku, B., et al., Testosterone Protects Against Severe Influenza by Reducing the Pro-Inflammatory Cytokine Response in the Murine Lung. Frontiers in immunology, 2020. 11: p. 697-697.

\section{Figures}


A

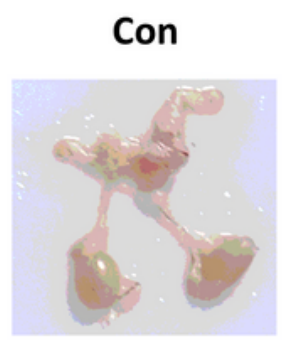

B

\section{ZIKV}

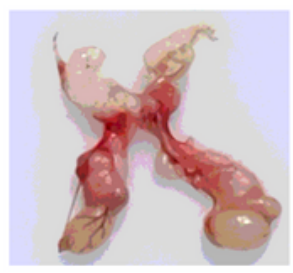

Con ZIKV

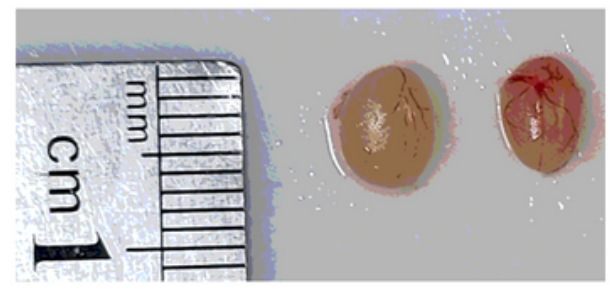

C

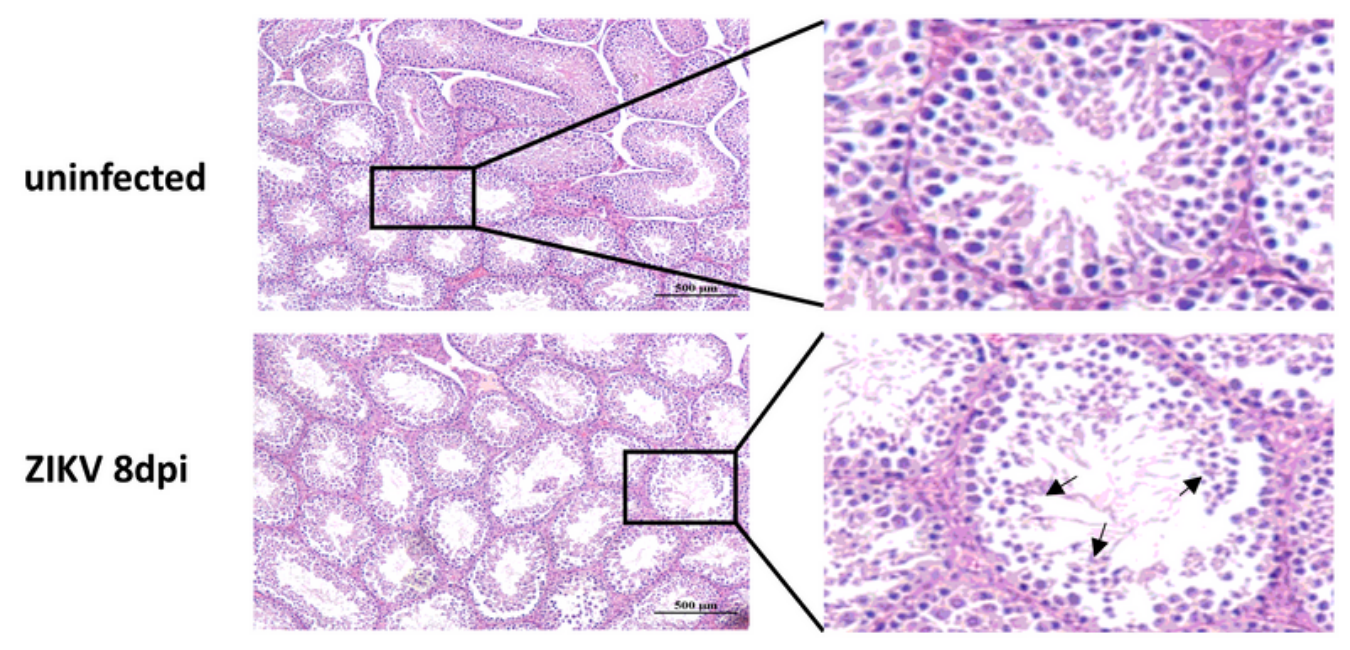

D

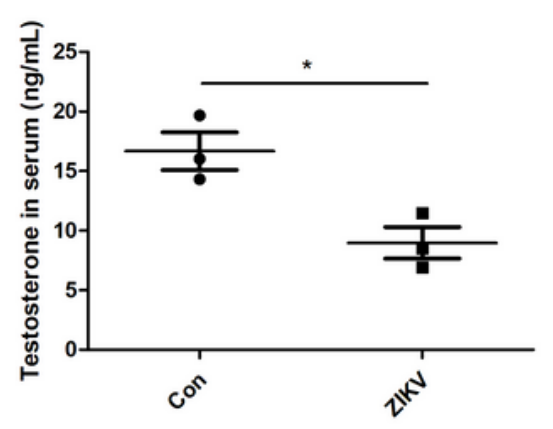

E

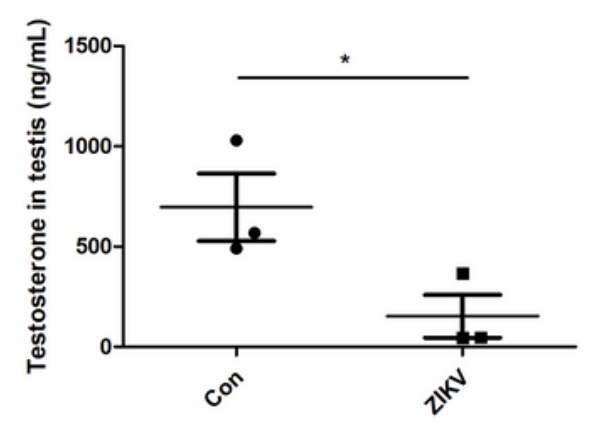

\section{Figure 1}

ZIKV infection caused testicular damage and decreased serum testosterone in mice. A-B Comparison of male reproductive system (A) and testicular appearance (B) between ZIKV-infected and -uninfected mice. $C$ Mouse testes samples were fixed in buffered formalin and processed for routine H\&E. After ZIKV infection, the cells in seminiferous tubules are disordered, with a large number of spermatogonial cells shedding in the middle of seminiferous tubules (black arrow). D-E The levels of testosterone in serum and 
testis of mice were detected by ELISA kit, data were expressed as mean \pm SEM of three independent experiments. ${ }^{*} \mathrm{P}<0.05$

A

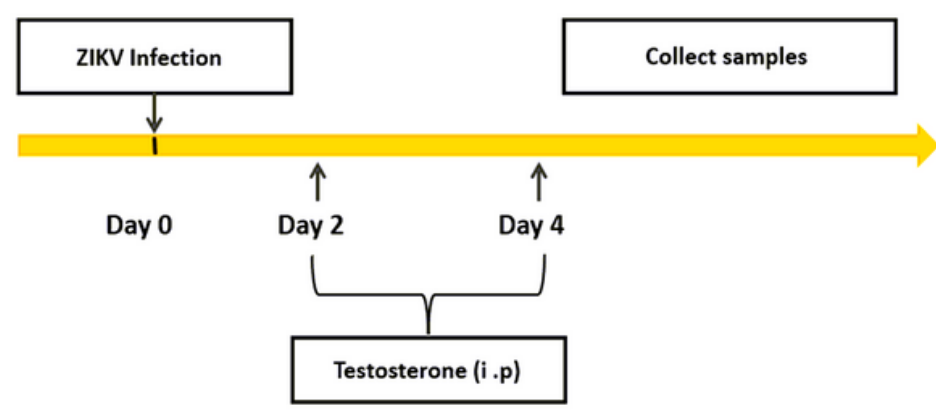

C

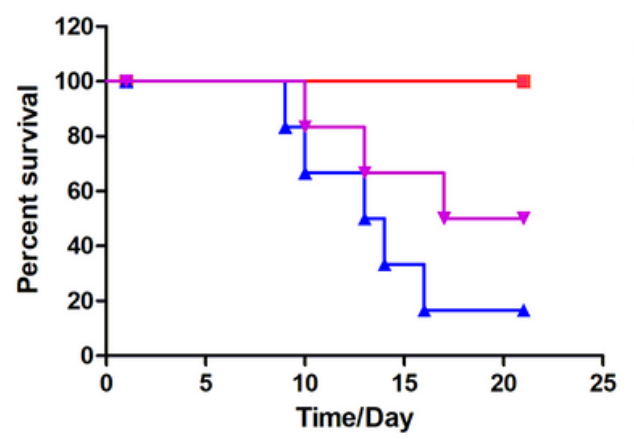

$\mathbf{E}$
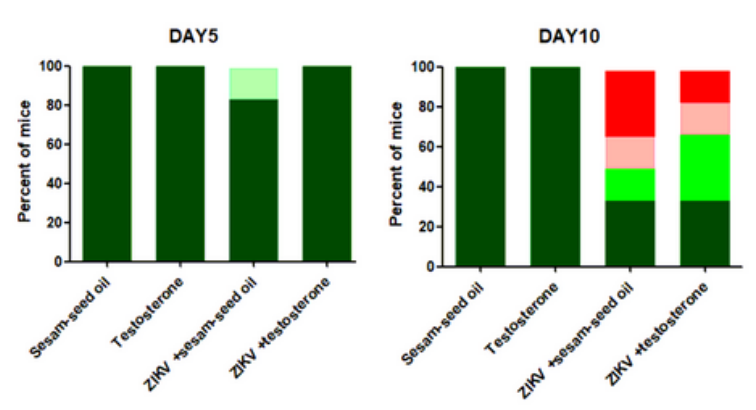

B

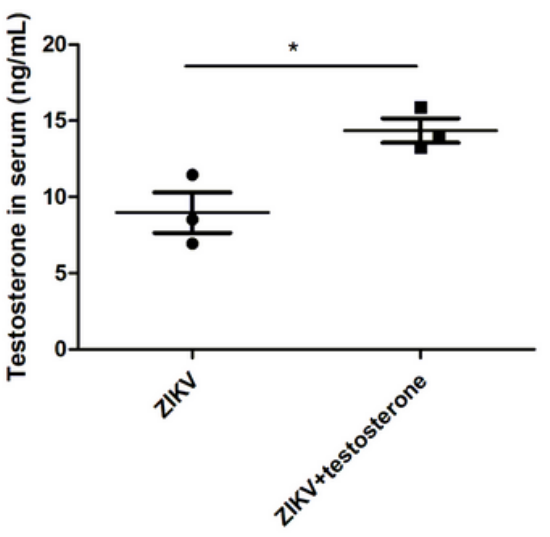

D

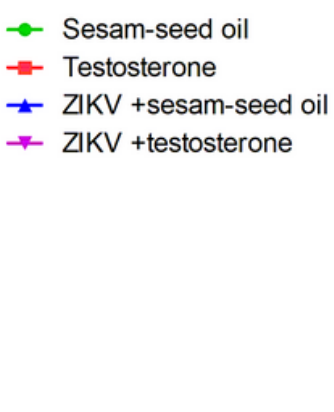

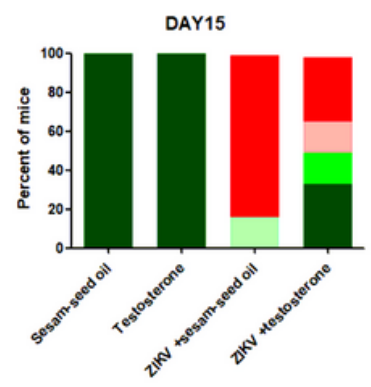

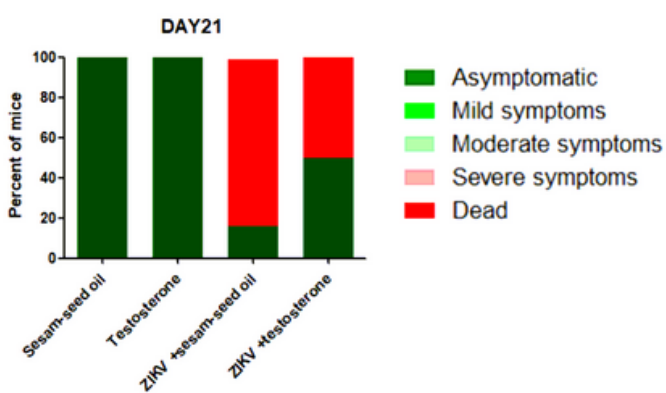

Figure 2

Testosterone plays a protective role during ZIKV infection in mice. A Diagram of experimental model in vivo. The testosterone treatment mode of ZIKV infected mice was described in the methods section. $B$ The testosterone concentration in serum of mice infected with ZIKV was detected by ELISA kit on 8 days post infection. C The survival curve of mice infected with ZIKV was monitored until day 21 post infection, $\mathrm{n}=10$ mice. $D$ Weight curve, the weight of each mouse divided by the average weight of each group on the first day. E Clinical signs of disease appeared in mice on the specified days after ZIKV infection. 
A

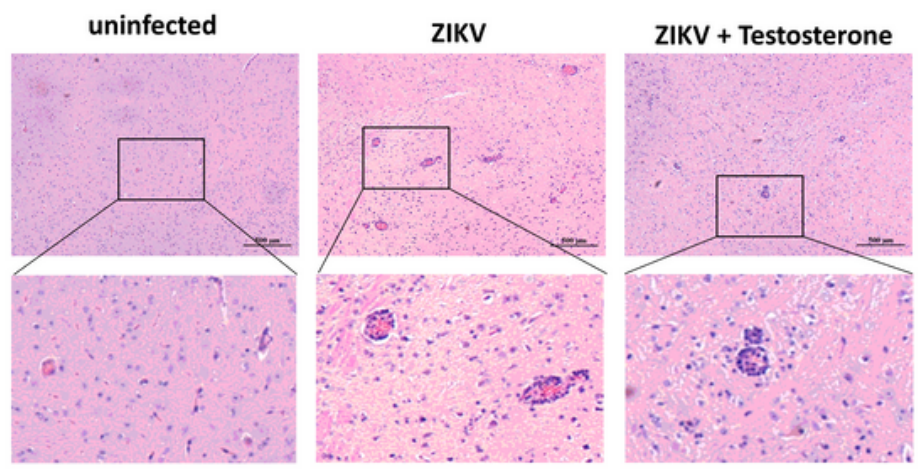

B
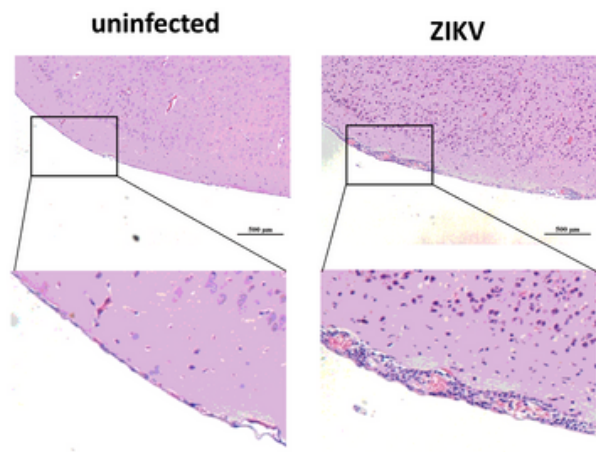

ZIKV + Testosterone

C
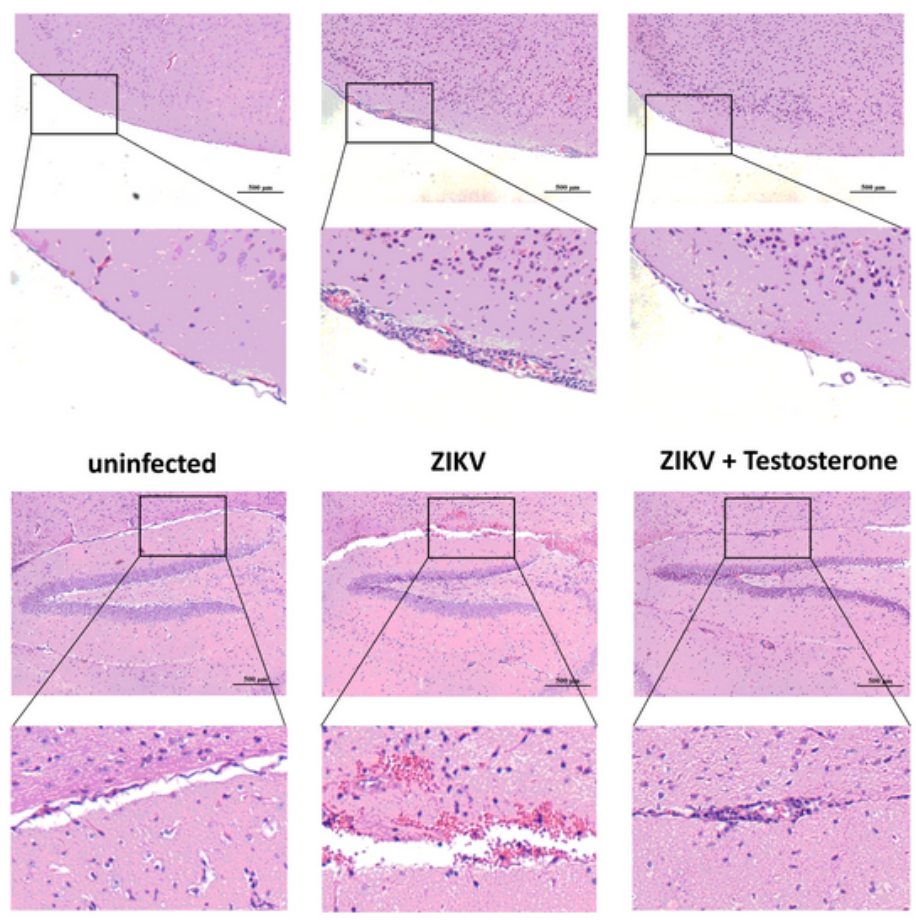

ZIKV + Testosterone

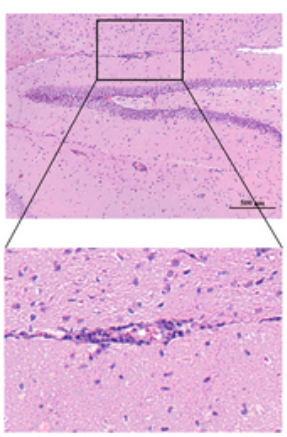

D

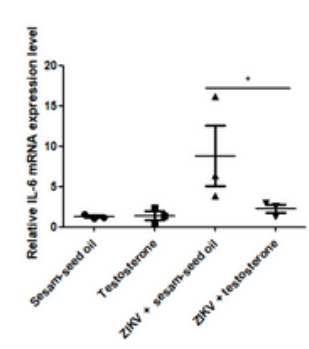

$\mathbf{E}$

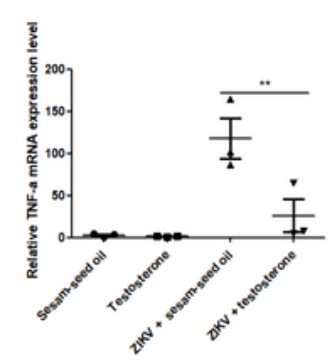

\section{Figure 3}

Testosterone treatment reduces ZIKV-induced inflammatory response in mouse brain tissues. A-C H\&E staining was performed to analyze the pathological changes of brain tissues. Testosterone treatment reduced the leaking out of inflammatory cells and perivascular cuffing forming $(A)$, attenuated the meningitis (B), and decreased the number of red blood cells around the hippocampus (C) in brain tissues 
of ZIKV infected mice. D-E The mRNA expression levels of inflammatory factors IL-6 (D) and TNF-a (E) were detected by real-time PCR. *P<0.05, **P<0.01

A
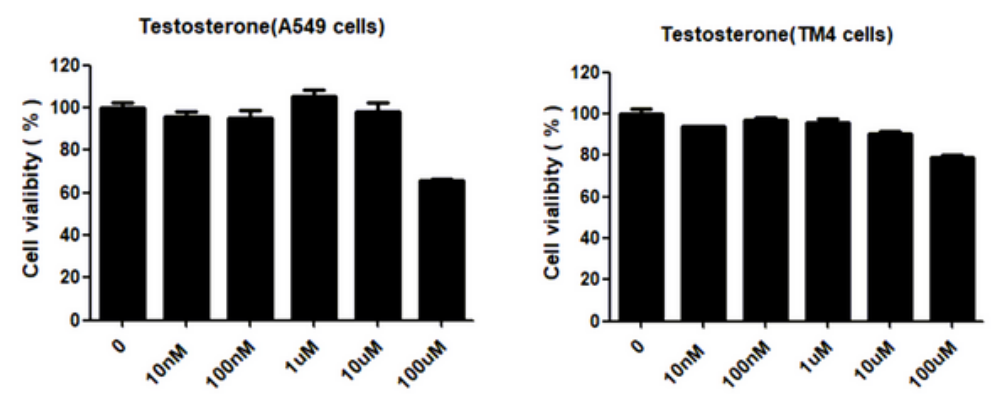

B
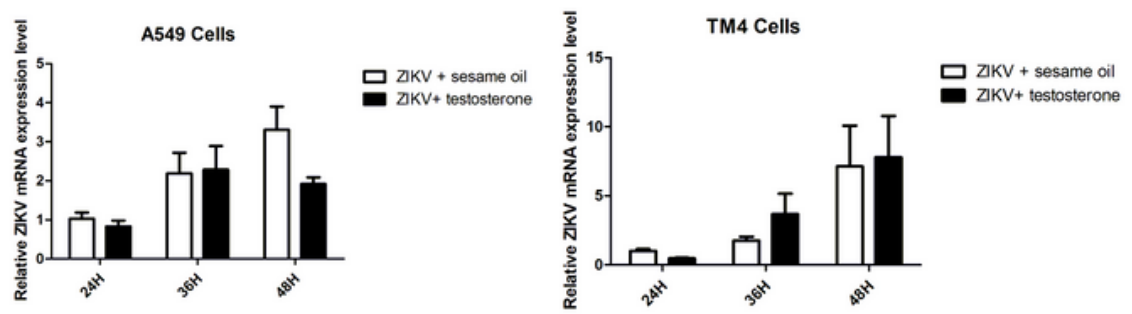

C
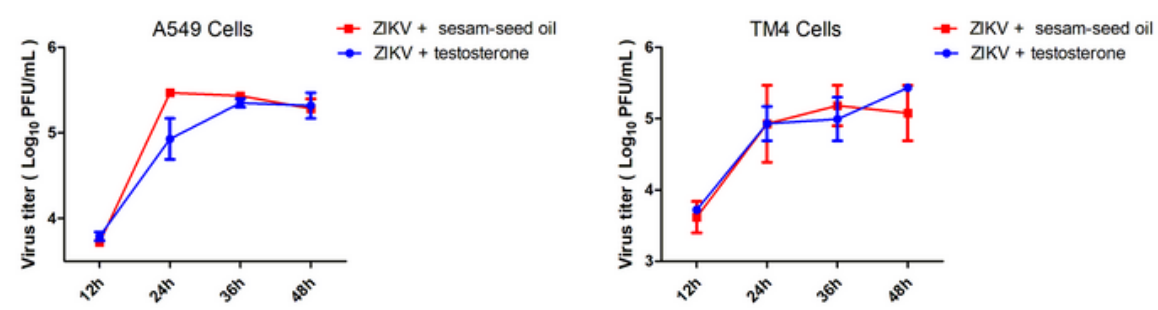

D

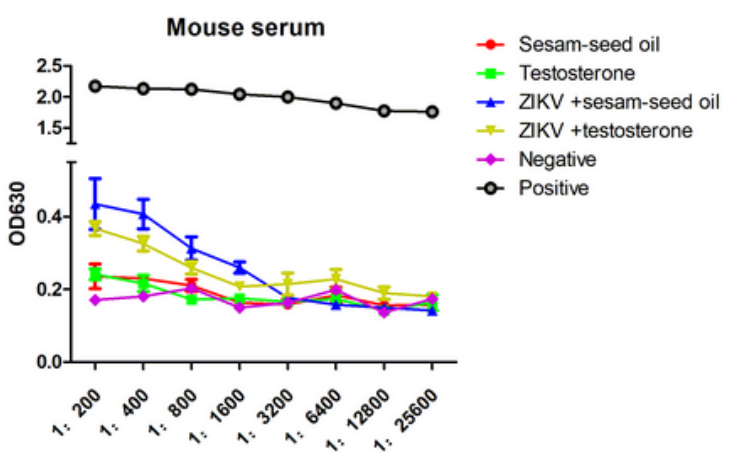

Figure 4

Testosterone does not affect viral replication and humoral immunity in ZIKV infected mice. A The toxicity of testosterone on A549 cells and TM4 cells was detected by using luminescence-based cell viability assay. B The expression level of ZIKV mRNA in A549 cells and TM4 cells after infection was detected by 
quantitative real-time PCR. C Virus plaque assay was used to detect the virus replication in A549 cells and TM4 cells infected with ZIKV. D Using ZIKV E protein as antigen, antibody levels in serum of mice were measured 8 days after viral infection.

A

Sesam-seed oil

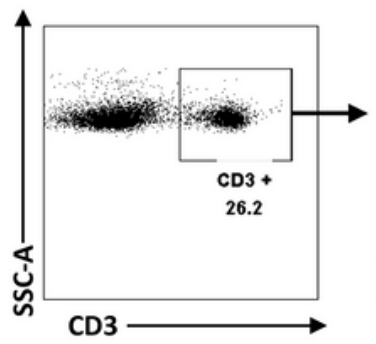

Testosterone

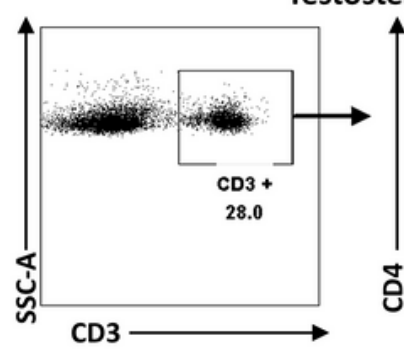

B

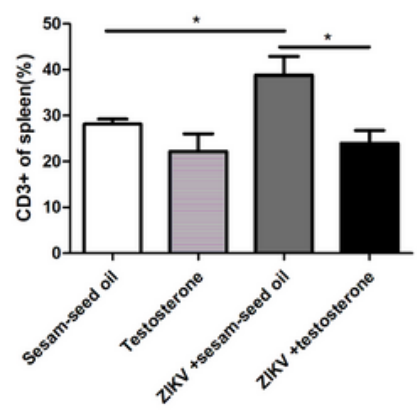

$\mathbf{E}$

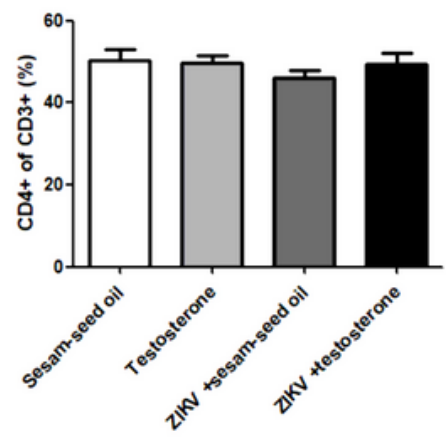

$\mathbf{F}$
ZIKV + Sesam-seed oil

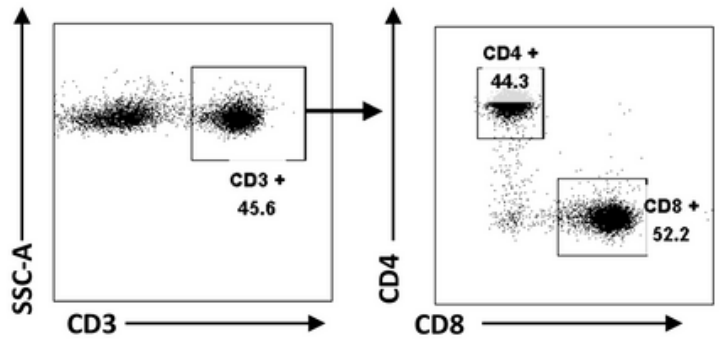

ZIKV + Testosterone

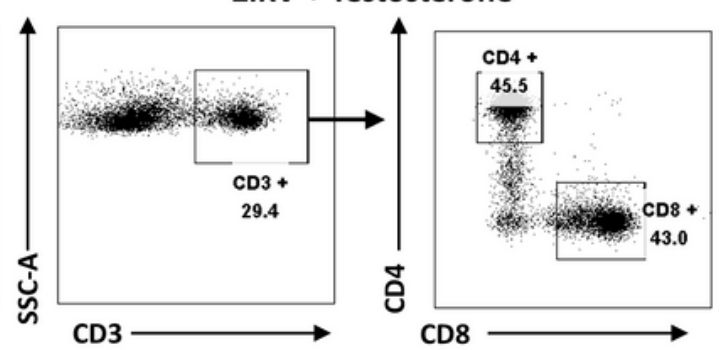

D
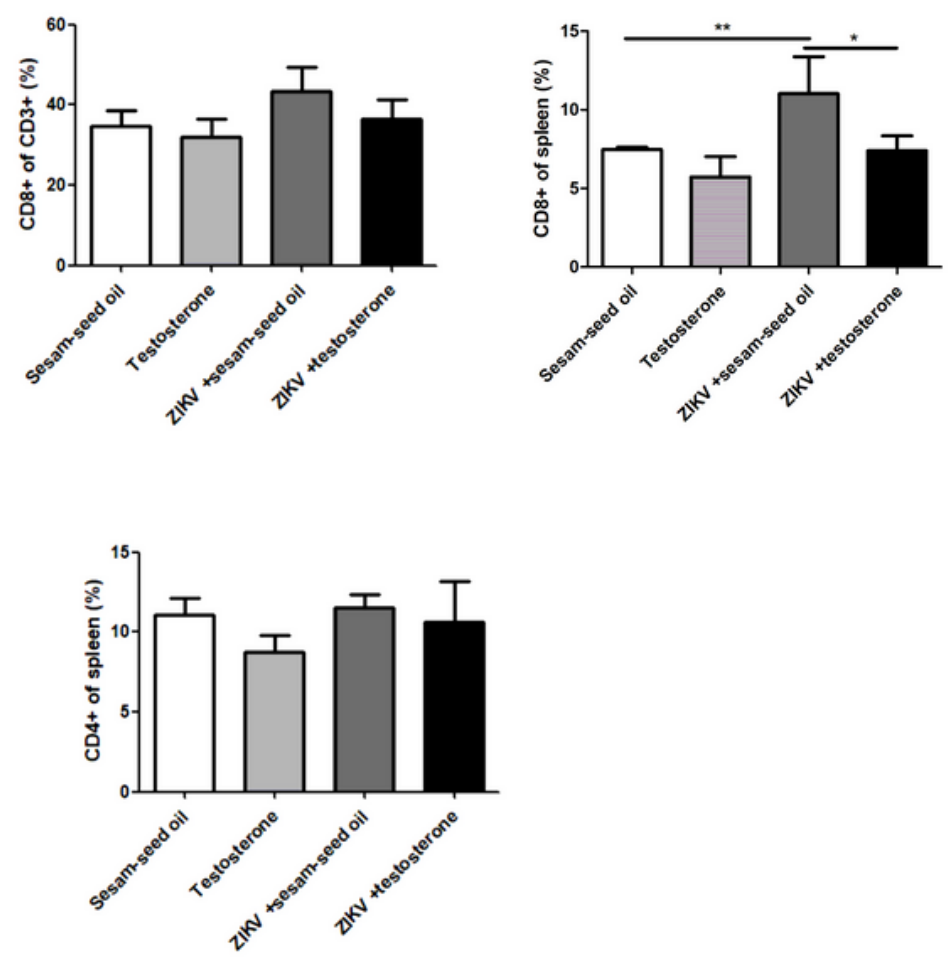

Figure 5

Testosterone inhibits T cell activation caused by ZIKV infection. A The activation proportion of CD3+ T cells CD 4+ T cells and CD 8+ T cells in spleen of mice in indicated groups were analyzed by flow 
cytometry. B Percentage of CD3+ T cells in spleen cells. $C, E$ the proportion of $C D 4+T$ cells and $C D 8+T$ cells in CD3+ T cells. D, F Percentage of CD4+ T cells and CD8+ $T$ cells in total spleen cells. ${ }^{*}<<0.05$, $\star * P<0.01$

A
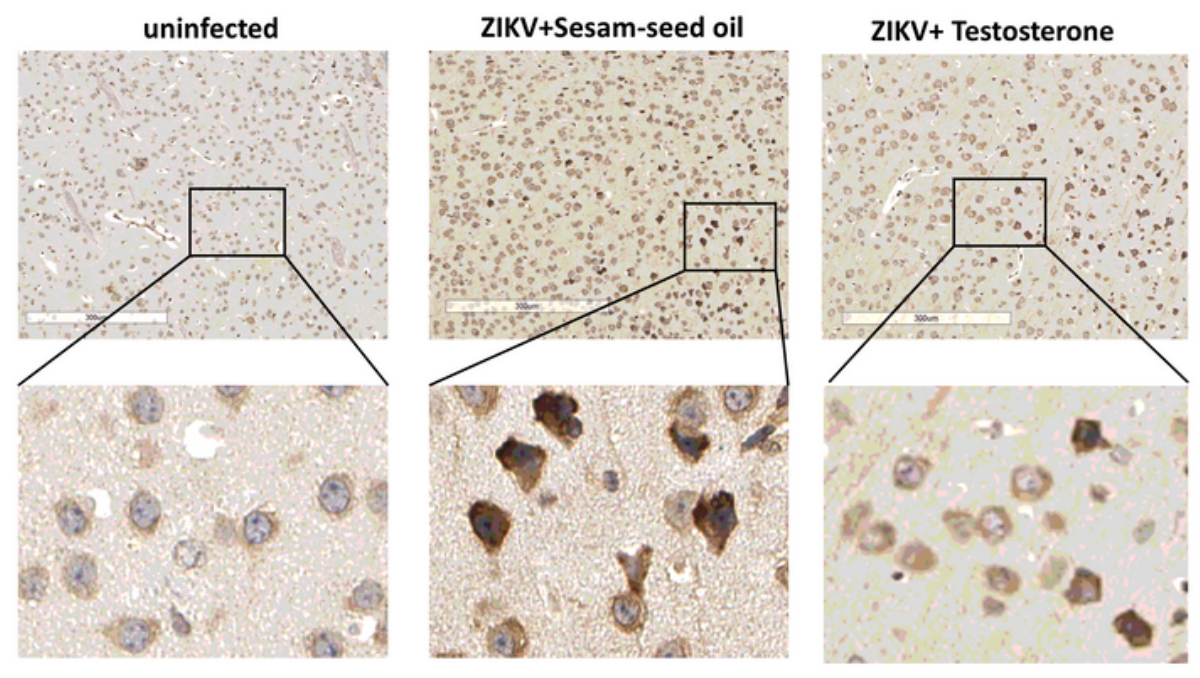

B

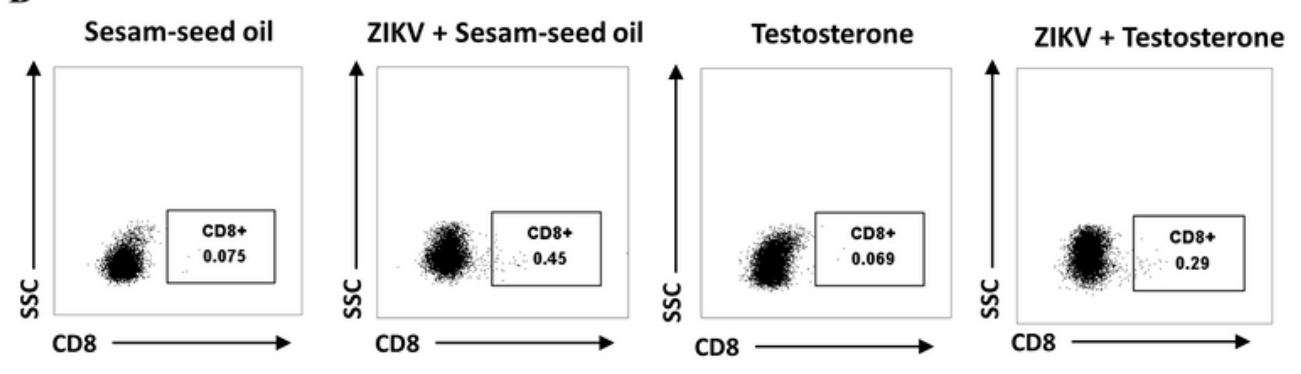

C

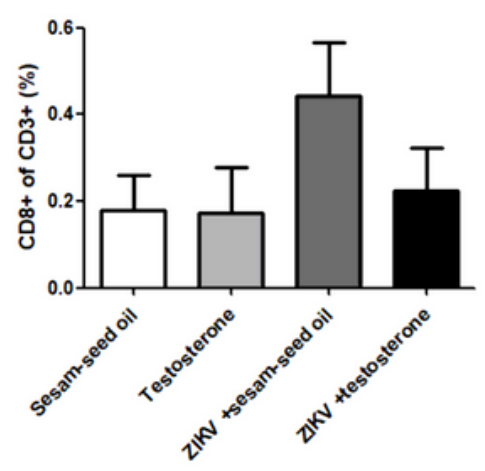

D

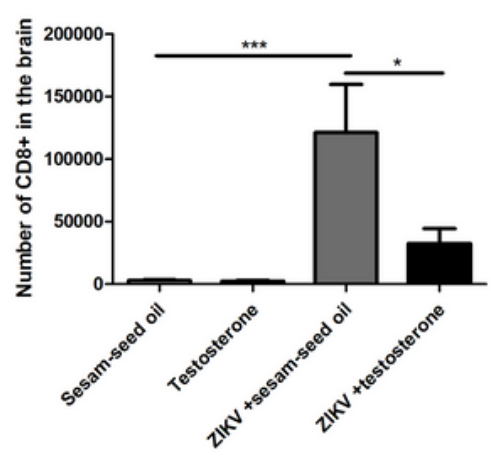

Figure 6

Testosterone treatment reduces the infiltration of CD8+ T cells in brain of ZIKV infected mice. A CD8+ T cells on mouse brain sections were detected by immunohistochemical assay. B Brain tissue samples 
were prepared and the number of CD8+ T cells in brain was determined by flow cytometry. C-D The ratio of CD8+ T cells in CD3+ T cells (C) and the number of CD8+ T cells in brain tissue (D) were calculated. ${ }^{*} P<0.05, * * P<0.01, * * * P<0.001$

A

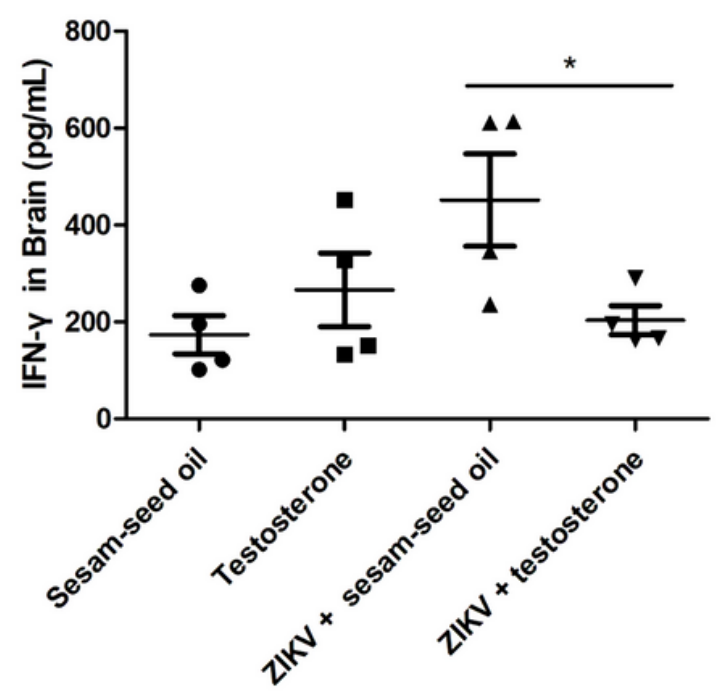

C

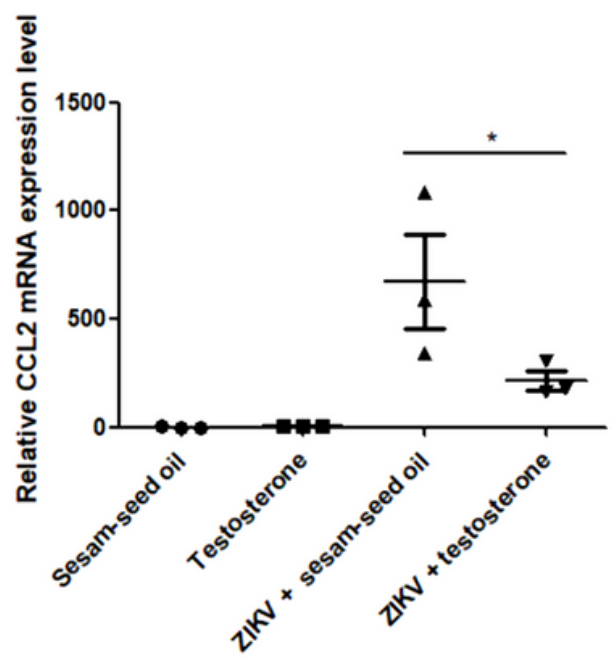

B

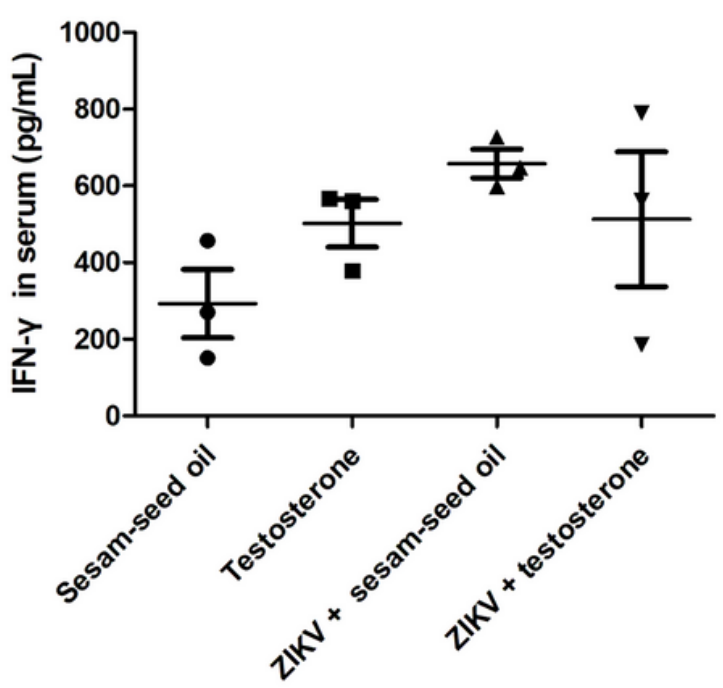

D

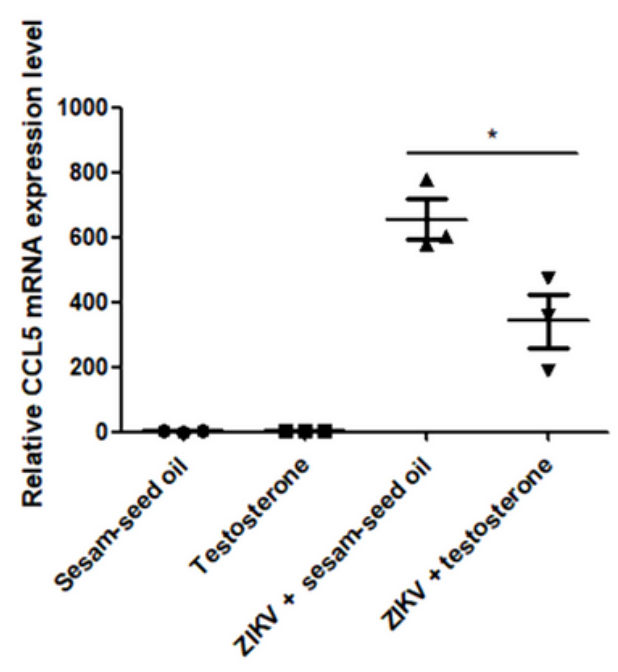

\section{Figure 7}

Testosterone treatment inhibits the expression of IFN- $y$ and chemokines in brain of ZIKV infected mice. A$B$ The protein levels of IFN- $\gamma$ in the brain (A) and serum (B) of mice in indicated groups were detected by ELISA. C-D The mRNA levels of chemokines CCL2 and CCL5 in brain of mice in indicated groups were detected by real-time RT-PCR. * $\mathrm{P}<0.05$ 